\title{
Simulations of future changes in thermal structure of Lake Erken: Proof of concept for ISIMIP2b lake sector local simulation strategy
}

Ana I. Ayala ${ }^{1,2}$, Simone Moras ${ }^{1}$, Don C. Pierson ${ }^{1}$

${ }^{1}$ Department of Ecology and Genetics, Limnology, Uppsala University, Uppsala, 752 36, Sweden

$5{ }^{2}$ Department of Applied Physics, Nonlinearity and Climate Group, University of Geneva, Geneva, CH-1211, Switzerland

Correspondence to: Ana I. Ayala (isabel.ayala. zamora@ebc.uu.se)
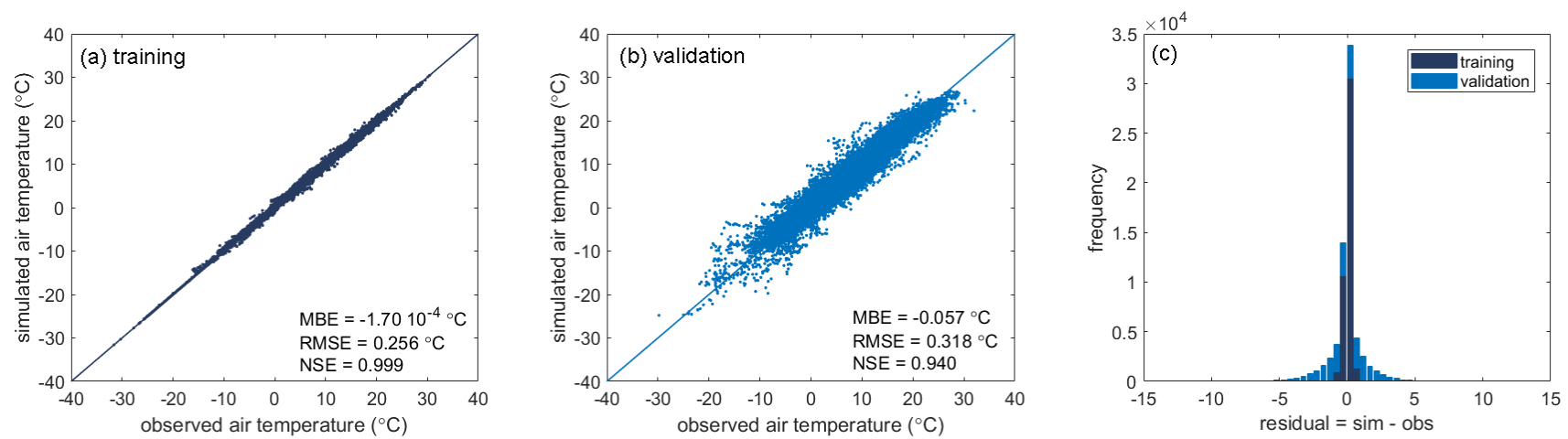

Figure S1: Measured vs predicted air temperature for the (a) training and (b) validation data sets, and (c) frequency distribution of air temperature residuals.
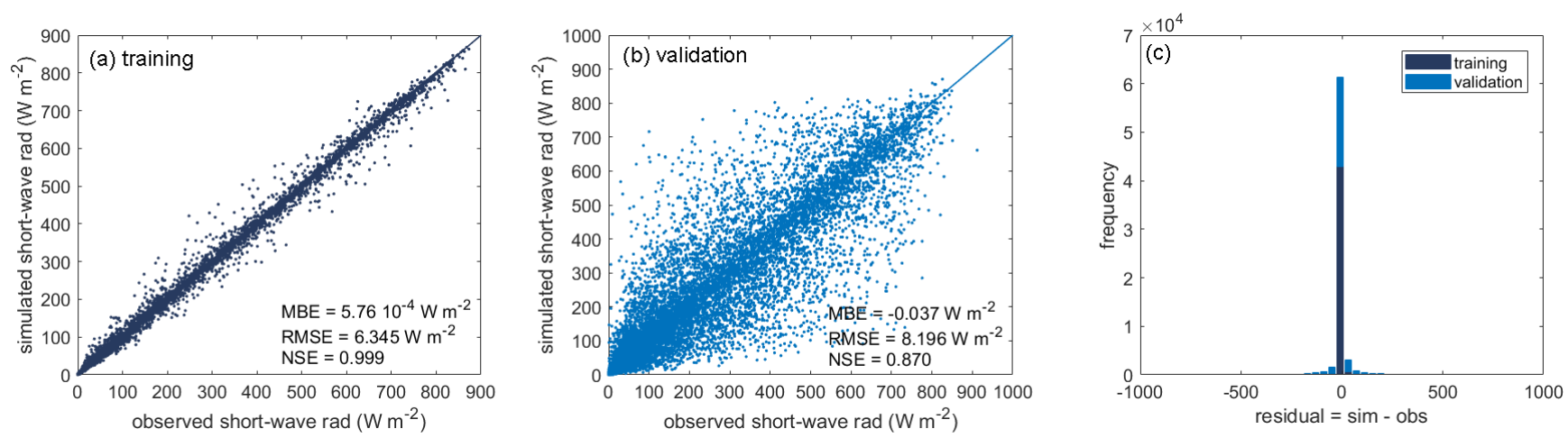

Figure S2: Measured vs predicted short-wave radiation for the (a) training and (b) validation data sets, and (c) frequency distribution of short-wave radiation residuals. 

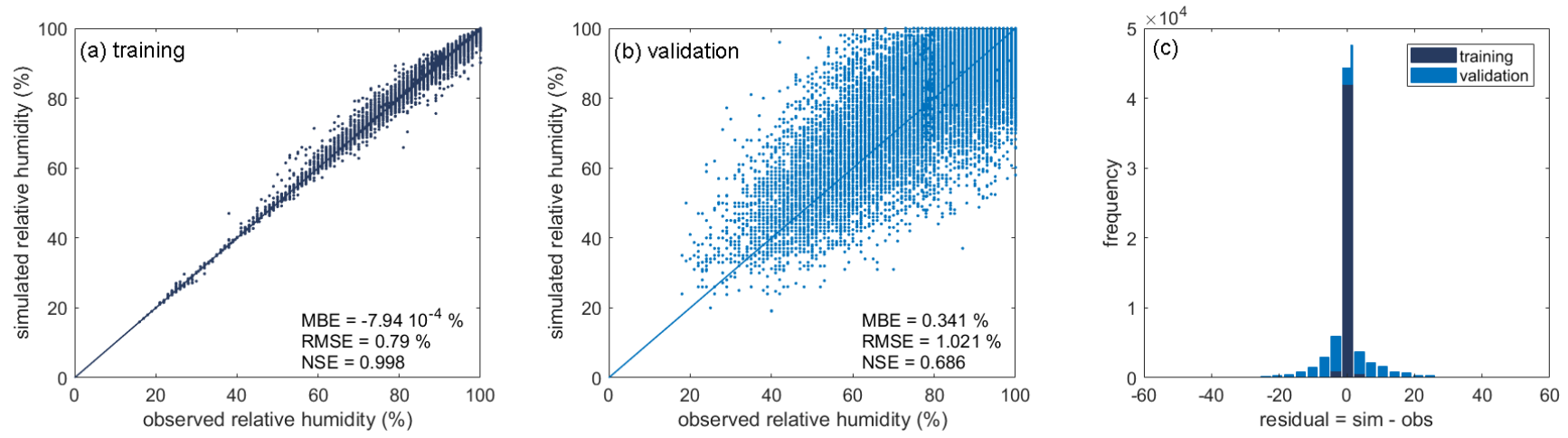

Figure S3: Measured vs predicted relative humidity for the (a) training and (b) validation data sets, and (c) frequency distribution 15 of relative humidity residuals.
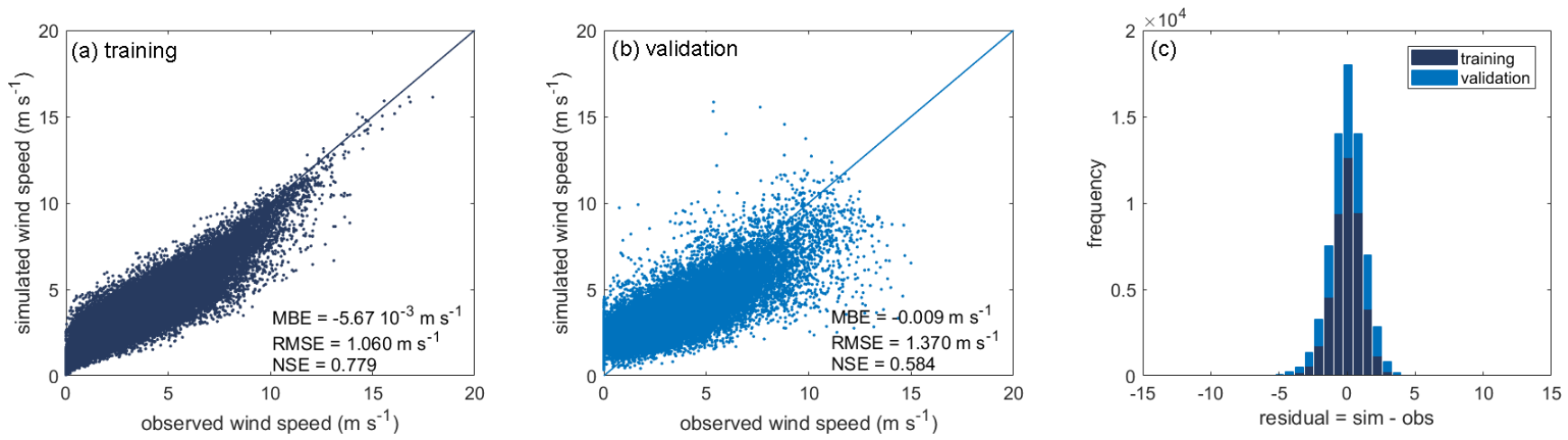

Figure S4: Measured vs predicted wind speed for the (a) training and (b) validation data sets, and (c) frequency distribution of wind speed residuals.

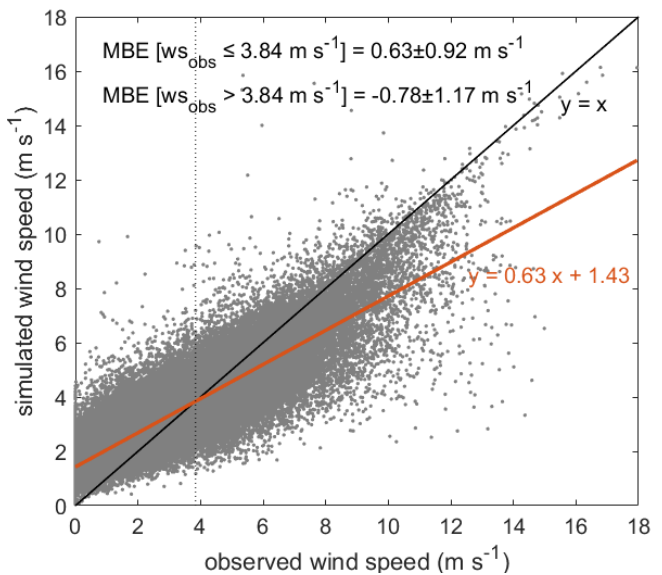

20 Figure S5: MBE of wind speed predictions using GRNN model. 

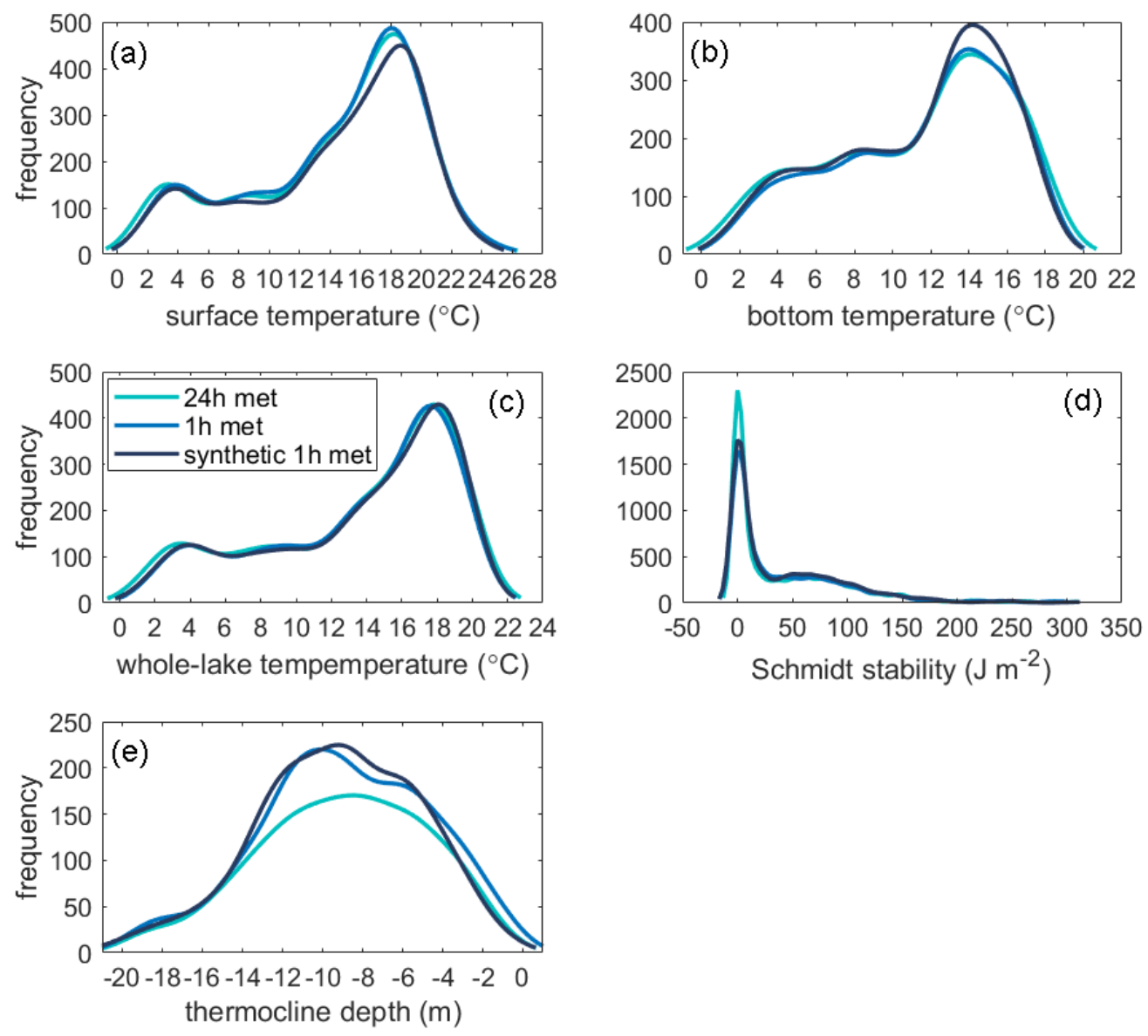

Figure S6: Daily averaged (a) surface temperature, (b) bottom temperature, (c) whole-lake temperature, (d) Schmidt stability and (e) thermocline depth from 2006 to 2016 (from April to September), showing results from the lake model forced with daily, hourly and synthetic hourly meteorological data. 

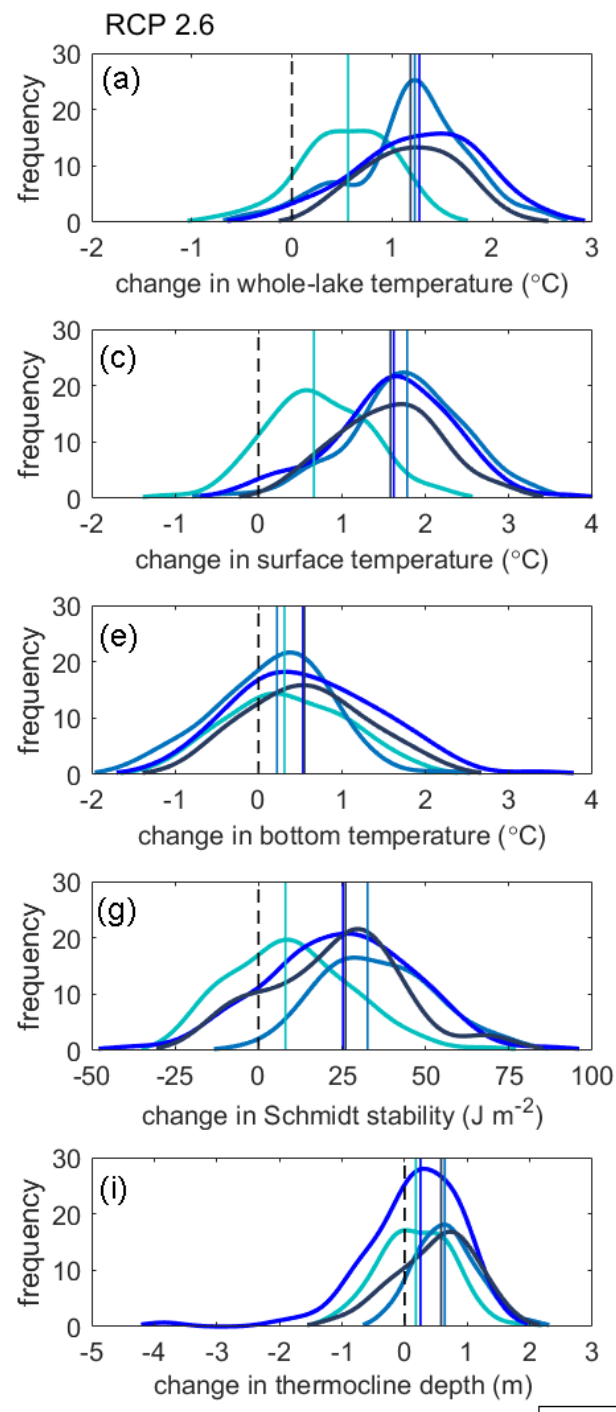
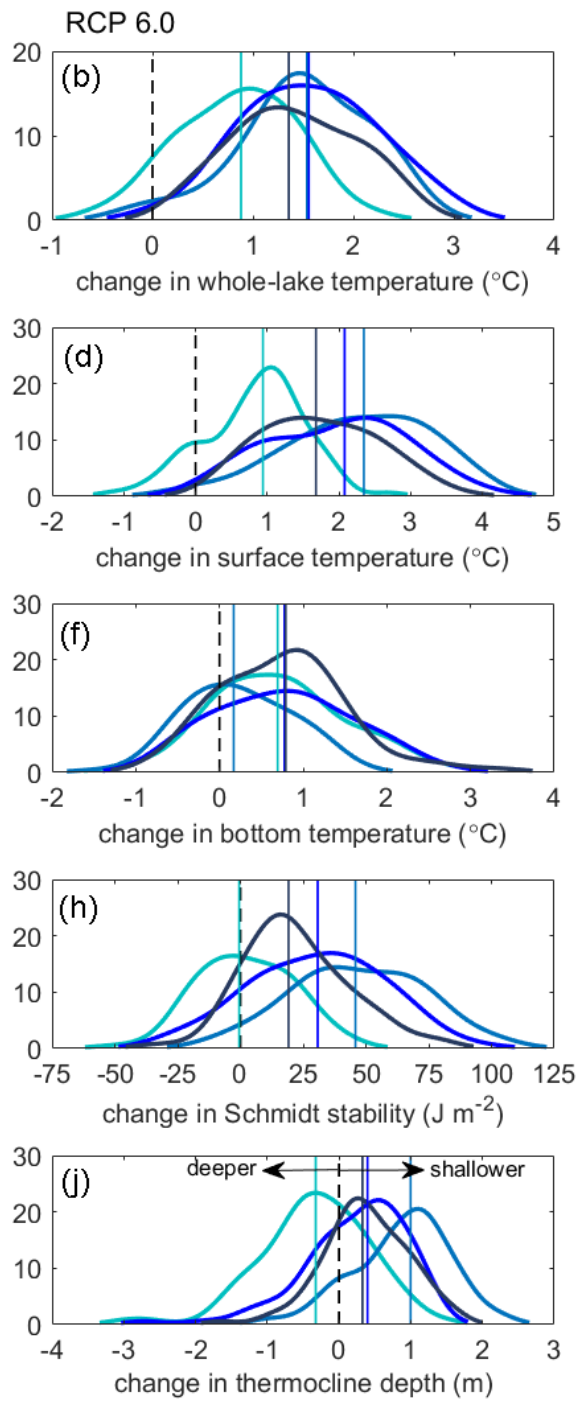

Figure S7: Changes in anomalies calculated from annually averaged (from April to September) (a)-(b) whole-lake temperature, (c)-(d) surface temperature, (e)-(f) bottom temperature, (g)-(h) Schmidt stability, (i)-(j) thermocline depth under (a)-(c)-(e)-(g)-(i) RCP 2.6 and (b)-(d)-(f)-(h)-(j) RCP 6.0, showing results from the lake model forced with hourly GFDL-ESM2M, HadGEM2-ES,

30 IPSL-CM5A-LR and MIROC5 projections. All changes are for 2006-2099 relative to 1975-2005. The median (vertical line) is also shown. 

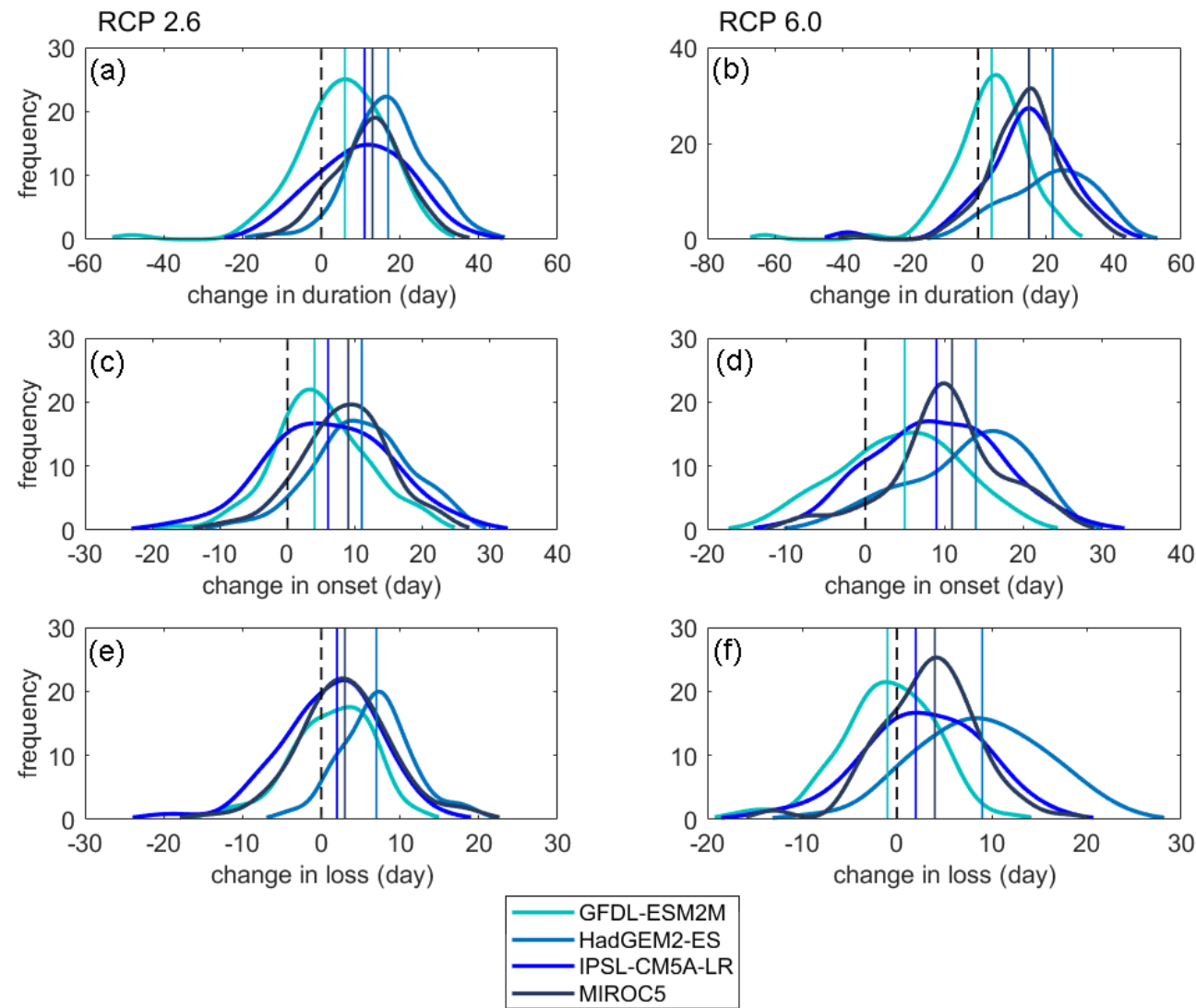

Figure S8: Changes in the calculated annual anomalies of the (a)-(b) duration, (c)-(d) onset and (e)-(f) loss of stratification under (a)-(c)-(e) RCP 2.6 and (b)-(d)-(f) RCP 6.0, showing results from the lake model forced with hourly GFDL-ESM2M, HadGEM2-

35 ES, IPSL-CM5A-LR and MIROC5 projections. All changes are for 2006-2099 relative to 1975-2005. The median (vertical line) is also shown. 

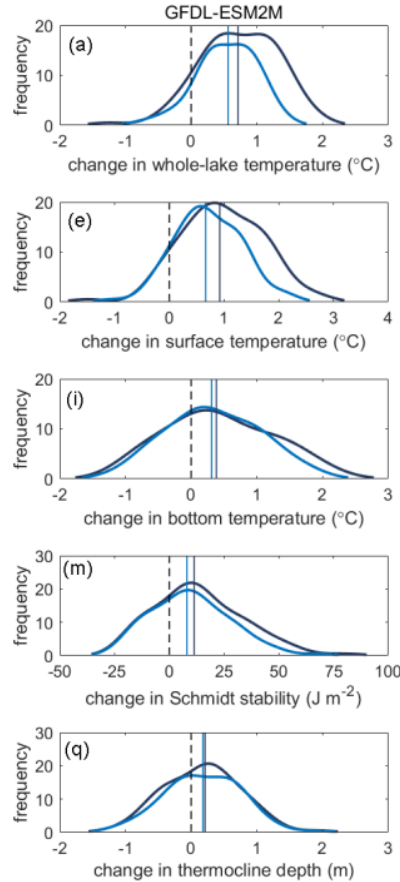
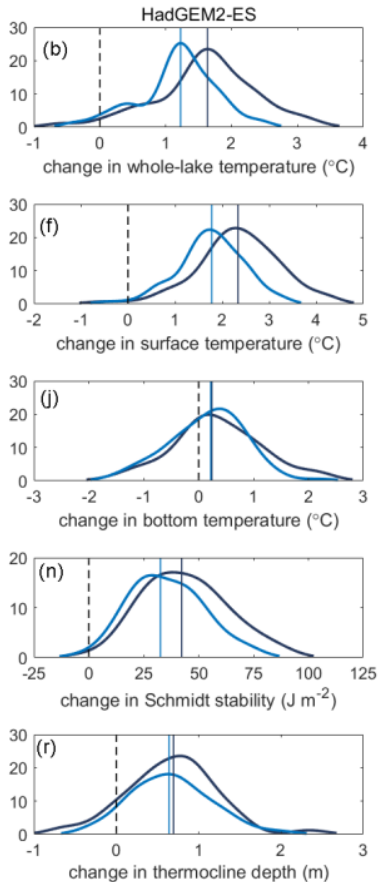
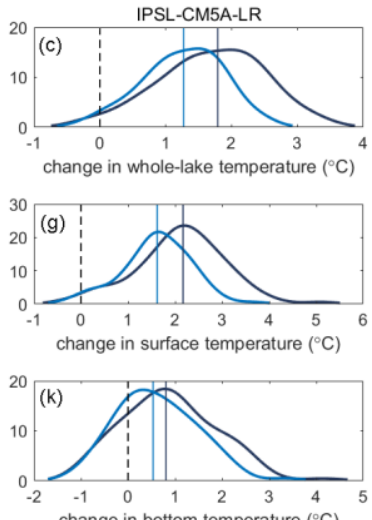

change in bottom temperature $\left({ }^{\circ} \mathrm{C}\right)$
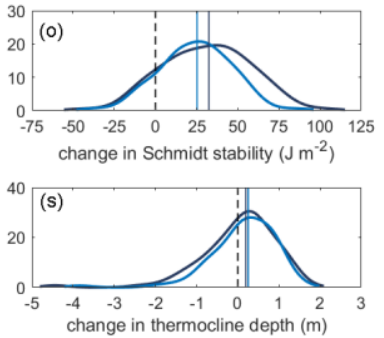
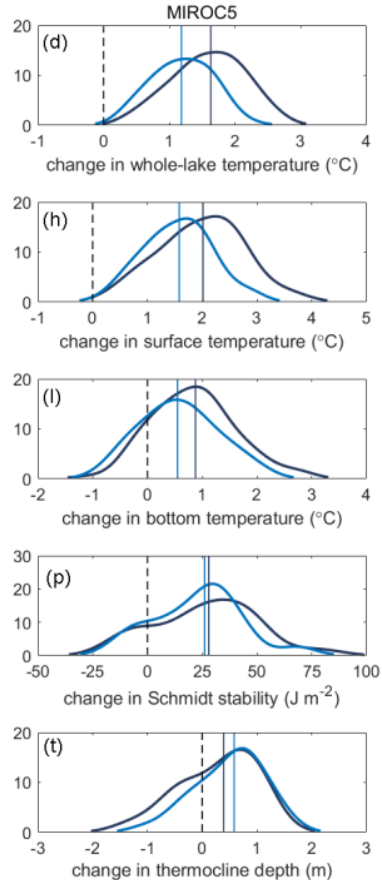

-24h met $1 \mathrm{~h}$ met

Figure S9: Changes in annually averaged (from April to September) (a)-(b)-(c)-(d) whole-lake temperature, (e)-(f)-(g)-(h) surface temperature, (i)-(j)-(k)-(l) bottom temperature, (m)-(n)-(o)-(p) Schmidt stability and (q)-(r)-(s)-(t) thermocline depth under RCP 40 2.6, showing results from the lake model forced with daily vs synthetic hourly GFDL-ESM2M, HadGEM2-ES, IPSL-CM5A-LR and MIROC5 projections. All changes are for 2006-2099 relative to 1975-2005. The mean (vertical line) is also shown. 

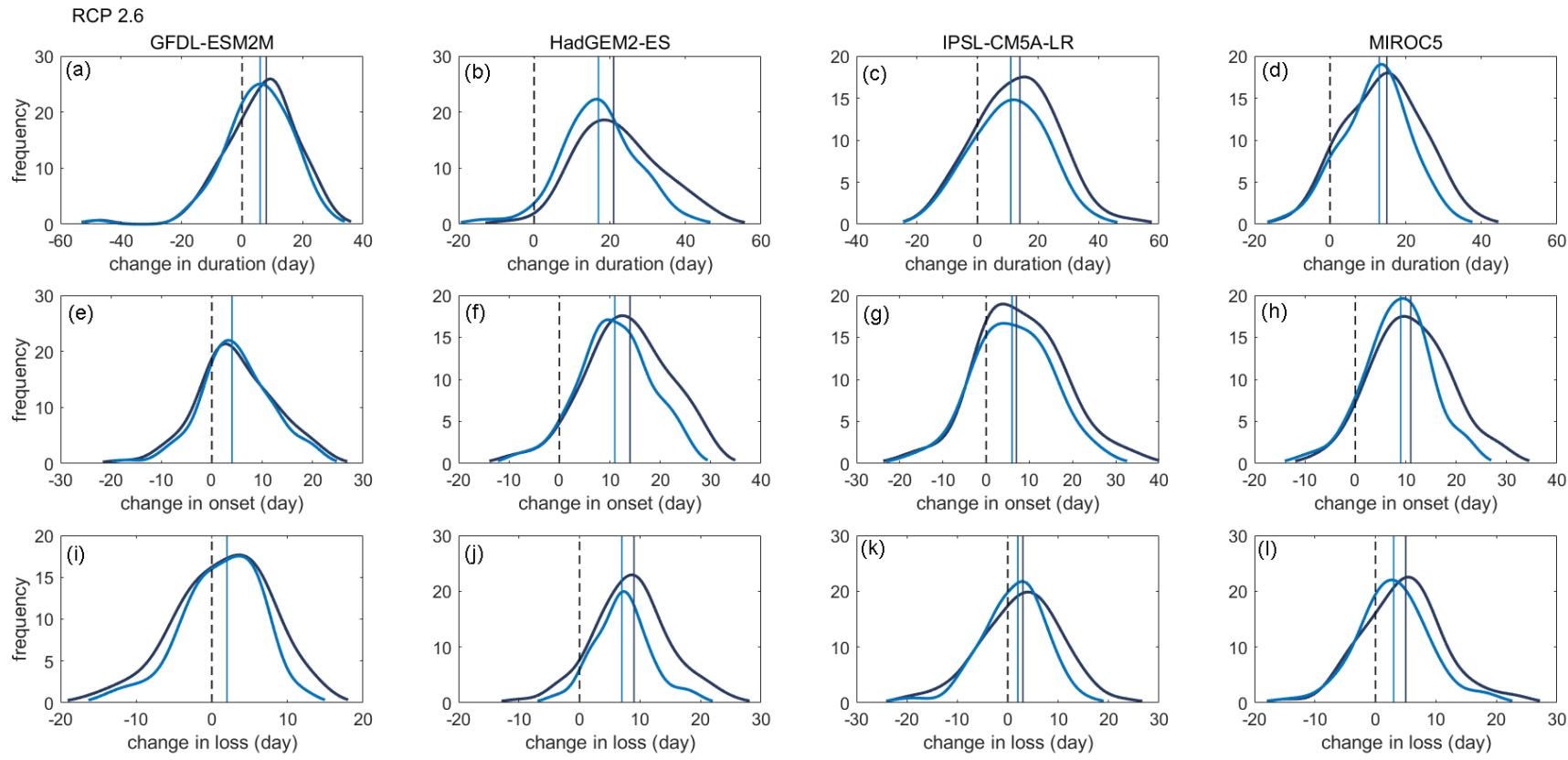

-24h met $1 \mathrm{~h}$ met

Figure S10: Changes in (a)-(b)-(c)-(d) duration, (e)-(f)-(g)-(h) onset and (i)-(j)-(k)-(l) loss of stratification under RCP 2.6, showing results from the lake model forced with daily vs synthetic hourly GFDL-ESM2M, HadGEM2-ES, IPSL-CM5A-LR and MIROC5 projections. All changes are for 2006-2099 relative to 1975-2005. The mean (vertical line) is also shown. 

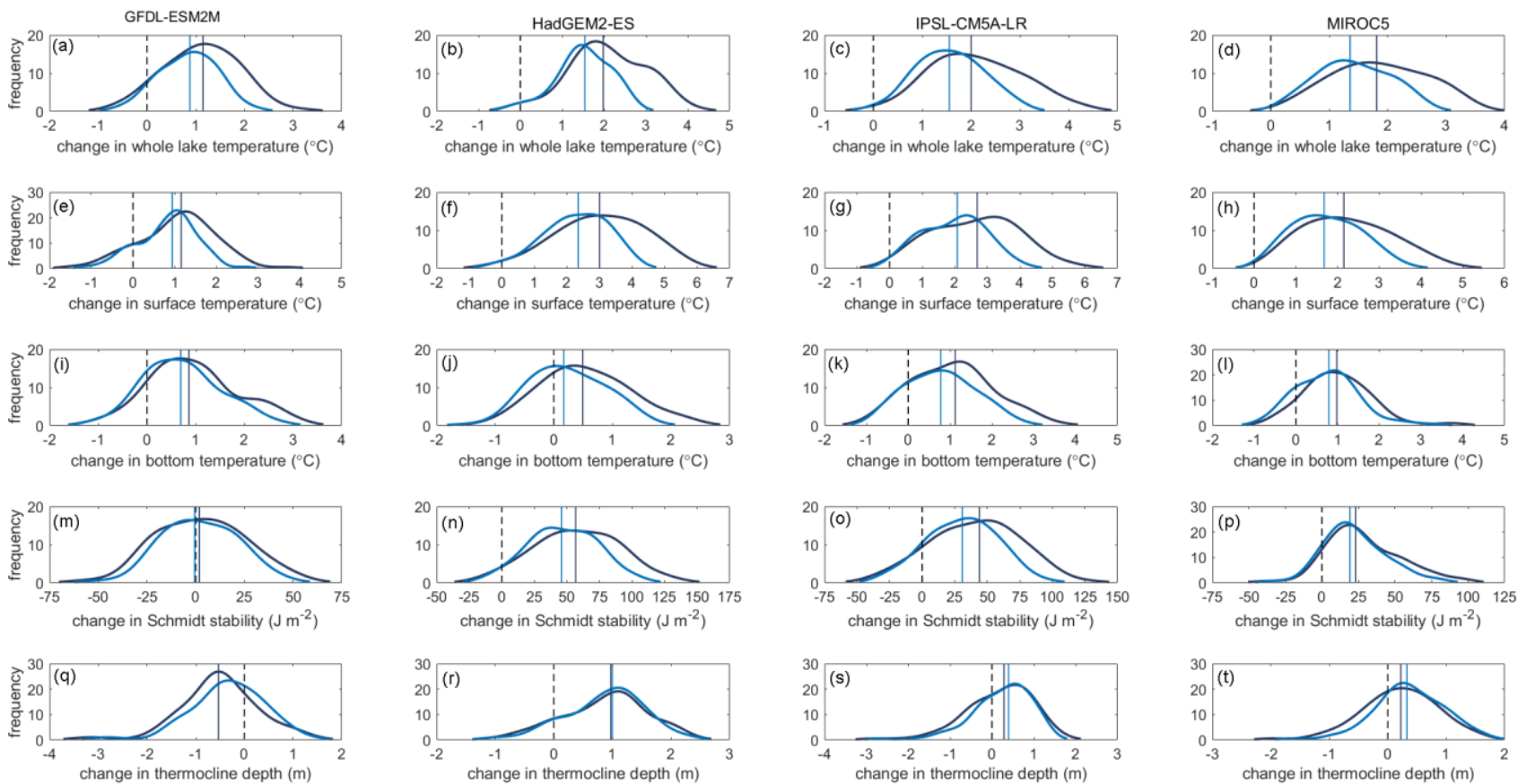

- $24 \mathrm{~h}$ met $1 \mathrm{~h}$ met

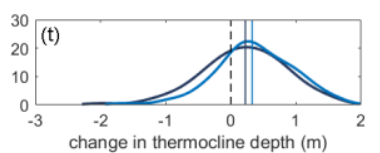

Figure S11: Changes in annually averaged (from April to September) (a)-(b)-(c)-(d) whole-lake temperature, (e)-(f)-(g)-(h) surface temperature, (i)-(j)-(k)-(l) bottom temperature, (m)-(n)-(o)-(p) Schmidt stability and (q)-(r)-(s)-(t) thermocline depth under RCP 6.0, showing results from the lake model forced with daily vs synthetic hourly GFDL-ESM2M, HadGEM2-ES, IPSL-CM5A-LR and MIROC5 projections. All changes are for 2006-2099 relative to 1975-2005. The mean (vertical line) is also shown. 

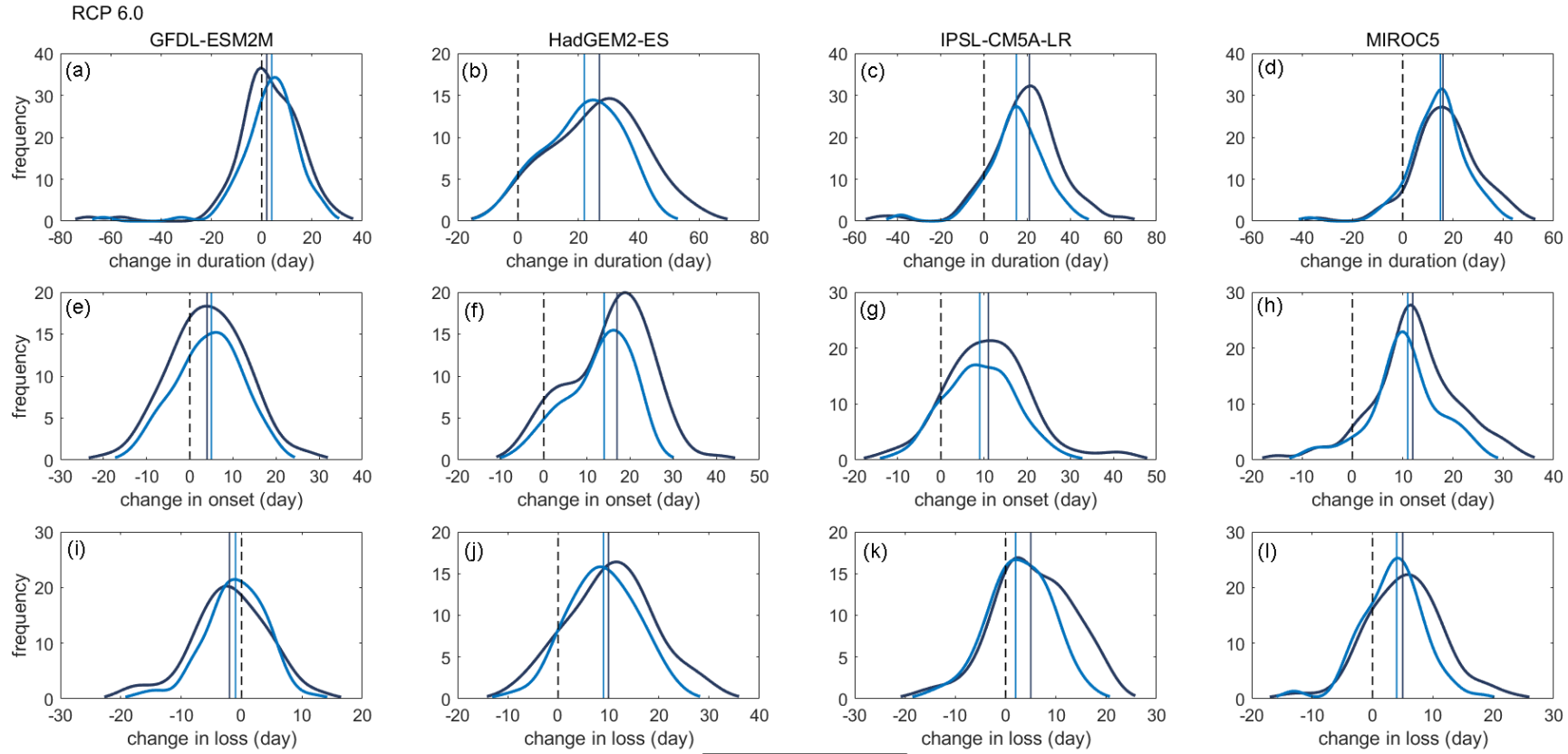

- 24h met $1 \mathrm{~h}$ met

Figure S12: Changes in (a)-(b)-(c)-(d) duration, (e)-(f)-(g)-(h) onset and (i)-(j)-(k)-(l) loss of stratification under RCP 6.0, showing results from the lake model forced with daily vs synthetic hourly GFDL-ESM2M, HadGEM2-ES, IPSL-CM5A-LR and MIROC5 projections. All changes are for 2006-2099 relative to 1975-2005. The mean (vertical line) is also shown. 
55 Table S1. Projected percentiles of change (2006-2099) in air temperature, surface temperature, bottom temperature, whole-lake temperature, Schmidt stability, thermocline depth, duration, onset and loss of stratification.

RCP 2.6

\section{GFDL-ESM2M}

\begin{tabular}{|c|c|c|c|c|c|c|c|c|c|c|c|c|c|c|}
\hline & \multicolumn{7}{|c|}{$24 \mathrm{~h}$ met } & \multicolumn{7}{|c|}{$1 \mathrm{~h}$ met } \\
\hline & P5 & P10 & $\mathrm{P} 25$ & P50 & P75 & P90 & P95 & P5 & P10 & $\mathrm{P} 25$ & P50 & P75 & P90 & P95 \\
\hline surface temp $\left({ }^{\circ} \mathrm{C}\right)$ & -0.21 & 0.01 & 0.47 & 0.92 & 1.55 & 1.87 & 2.17 & -0.25 & -0.06 & 0.29 & 0.66 & 1.17 & 1.42 & 1.59 \\
\hline bottom temp $\left({ }^{\circ} \mathrm{C}\right)$ & -0.79 & -0.71 & -0.10 & 0.38 & 1.08 & 1.54 & 1.79 & -0.74 & -0.59 & -0.16 & 0.31 & 0.87 & 1.24 & 1.55 \\
\hline whole-lake temp $\left({ }^{\circ} \mathrm{C}\right)$ & -0.20 & -0.05 & 0.33 & 0.71 & 1.18 & 1.46 & 1.60 & -0.29 & -0.06 & 0.26 & 0.56 & 0.88 & 1.10 & 1.18 \\
\hline Schmidt stability $\left(\mathrm{J} \mathrm{m}^{-2}\right)$ & -14.96 & -13.63 & 0.66 & 11.30 & 25.76 & 39.70 & 49.86 & -16.30 & -13.10 & -3.88 & 8.07 & 21.31 & 31.71 & 42.98 \\
\hline thermocline depth (m) & -0.77 & -0.54 & -0.27 & 0.21 & 0.57 & 0.91 & 1.08 & -0.76 & -0.56 & -0.22 & 0.18 & 0.60 & 0.83 & 1.10 \\
\hline duration (days) & -14 & -8 & 0 & 8 & 13 & 21 & 23 & -14 & -8 & -1 & 6 & 13 & 19 & 20 \\
\hline onset (days) & -8 & -3 & 0 & 4 & 10 & 15 & 18 & -7 & -3 & 0 & 4 & 9 & 13 & 18 \\
\hline loss (days) & -10 & -6 & -2 & 2 & 6 & 9 & 12 & -8 & -5 & -2 & 2 & 5 & 7 & 8 \\
\hline
\end{tabular}

HadGEM2-ES

$24 \mathrm{~h}$ met

1h met

\begin{tabular}{|c|c|c|c|c|c|c|c|c|c|c|c|c|c|c|}
\hline & & & & $24 \mathrm{n}$ met & & & & & & & in met & & & \\
\hline & P5 & P10 & $\mathrm{P} 25$ & P50 & P75 & P90 & P95 & P5 & P10 & $\mathrm{P} 25$ & P50 & P75 & P90 & P95 \\
\hline surface temp $\left({ }^{\circ} \mathrm{C}\right)$ & 0.83 & 1.26 & 1.82 & 2.34 & 2.91 & 3.38 & 3.67 & 0.58 & 0.81 & 1.38 & 1.78 & 2.21 & 2.57 & 2.87 \\
\hline bottom temp $\left({ }^{\circ} \mathrm{C}\right)$ & -1.06 & -0.67 & -0.11 & 0.24 & 0.87 & 1.39 & 1.97 & -1.09 & -0.81 & -0.32 & 0.22 & 0.64 & 0.98 & 1.16 \\
\hline whole-lake temp $\left({ }^{\circ} \mathrm{C}\right)$ & 0.21 & 0.58 & 1.31 & 1.64 & 2.09 & 2.42 & 2.94 & 0.07 & 0.35 & 0.96 & 1.23 & 1.54 & 1.85 & 2.07 \\
\hline Schmidt stability $\left(\mathrm{J} \mathrm{m}^{-2}\right)$ & 15.24 & 20.66 & 29.33 & 42.24 & 55.61 & 70.42 & 79.13 & 9.95 & 15.46 & 22.78 & 32.64 & 46.23 & 56.88 & 64.88 \\
\hline thermocline depth (m) & -0.19 & -0.03 & 0.33 & 0.70 & 1.00 & 1.30 & 1.46 & -0.13 & 0.15 & 0.31 & 0.64 & 0.99 & 1.29 & 1.46 \\
\hline duration (days) & 8 & 10 & 14 & 21 & 29 & 38 & 43 & 0 & 6 & 11 & 17 & 22 & 30 & 32 \\
\hline onset (days) & 0 & 3 & 9 & 14 & 19 & 25 & 27 & -1 & 2 & 7 & 11 & 15 & 21 & 23 \\
\hline loss (days) & -3 & 2 & 5 & 9 & 12 & 16 & 20 & 1 & 1 & 4 & 7 & 10 & 12 & 17 \\
\hline
\end{tabular}

IPSL-CM5A-LR

\begin{tabular}{|c|c|c|c|c|c|c|c|c|c|c|c|c|c|c|}
\hline & \multicolumn{7}{|c|}{$24 \mathrm{~h}$ met } & \multicolumn{7}{|c|}{$1 \mathrm{~h}$ met } \\
\hline & P5 & P10 & $\mathrm{P} 25$ & P50 & P75 & P90 & P95 & P5 & $\mathrm{P} 10$ & $\mathrm{P} 25$ & P50 & P75 & P90 & P95 \\
\hline surface temp $\left({ }^{\circ} \mathrm{C}\right)$ & 0.41 & 0.89 & 1.56 & 2.17 & 2.70 & 3.17 & 3.56 & 0.27 & 0.61 & 1.21 & 1.62 & 2.12 & 2.46 & 2.68 \\
\hline bottom temp $\left({ }^{\circ} \mathrm{C}\right)$ & -0.67 & -0.40 & 0.10 & 0.80 & 1.51 & 2.25 & 2.43 & -0.63 & -0.53 & 0.00 & 0.53 & 1.15 & 1.72 & 2.05 \\
\hline whole-lake temp $\left({ }^{\circ} \mathrm{C}\right)$ & 0.38 & 0.64 & 1.18 & 1.79 & 2.25 & 2.60 & 2.97 & 0.14 & 0.43 & 0.87 & 1.27 & 1.71 & 1.94 & 2.14 \\
\hline Schmidt stability $\left(\mathrm{J} \mathrm{m}^{-2}\right)$ & -10.81 & -4.88 & 12.79 & 32.41 & 48.64 & 64.18 & 72.57 & -10.87 & -6.31 & 11.04 & 25.26 & 38.61 & 51.46 & 56.70 \\
\hline
\end{tabular}




\begin{tabular}{|c|c|c|c|c|c|c|c|c|c|c|c|c|c|c|}
\hline thermocline depth (m) & -1.36 & -1.12 & -0.38 & 0.19 & 0.63 & 1.12 & 1.24 & -1.10 & -0.77 & -0.24 & 0.25 & 0.75 & 0.99 & 1.10 \\
\hline duration (days) & -8 & -6 & 4 & 14 & 21 & 29 & 32 & -10 & -5 & 1 & 11 & 19 & 25 & 26 \\
\hline onset (days) & -6 & -2 & 1 & 7 & 15 & 19 & 25 & -7 & -2 & 0 & 6 & 13 & 16 & 21 \\
\hline \multirow[t]{4}{*}{ loss (days) } & -12 & -7 & -2 & 3 & 8 & 12 & 15 & -10 & -7 & -2 & 2 & 5 & 8 & 11 \\
\hline & \multicolumn{14}{|c|}{ MIROC5 } \\
\hline & \multicolumn{7}{|c|}{$24 \mathrm{~h}$ met } & \multicolumn{7}{|c|}{$1 \mathrm{~h}$ met } \\
\hline & P5 & P10 & $\mathrm{P} 25$ & P50 & P75 & P90 & P95 & P5 & P10 & $\mathrm{P} 25$ & P50 & P75 & P90 & P95 \\
\hline surface temp $\left({ }^{\circ} \mathrm{C}\right)$ & 0.70 & 0.87 & 1.49 & 2.01 & 2.52 & 2.86 & 3.26 & 0.52 & 0.72 & 1.11 & 1.58 & 1.97 & 2.33 & 2.66 \\
\hline bottom temp $\left({ }^{\circ} \mathrm{C}\right)$ & -0.28 & -0.14 & 0.33 & 0.88 & 1.30 & 1.83 & 2.14 & -0.54 & -0.37 & 0.05 & 0.55 & 1.05 & 1.58 & 1.86 \\
\hline whole-lake temp $\left({ }^{\circ} \mathrm{C}\right)$ & 0.63 & 0.85 & 1.25 & 1.63 & 2.02 & 2.30 & 2.41 & 0.40 & 0.58 & 0.86 & 1.18 & 1.56 & 1.75 & 1.85 \\
\hline Schmidt stability $\left(\mathrm{J} \mathrm{m}^{-2}\right)$ & -10.47 & -6.77 & 10.73 & 27.97 & 42.59 & 52.39 & 66.87 & -10.34 & -6.82 & 8.72 & 26.08 & 35.17 & 45.22 & 62.77 \\
\hline thermocline depth $(\mathrm{m})$ & -1.08 & -0.71 & -0.26 & 0.39 & 0.85 & 1.06 & 1.17 & -0.63 & -0.40 & 0.08 & 0.58 & 0.92 & 1.27 & 1.40 \\
\hline duration (days) & -3 & 2 & 7 & 15 & 21 & 27 & 30 & -3 & 0 & 7 & 13 & 17 & 23 & 27 \\
\hline onset (days) & -2 & 2 & 6 & 11 & 16 & 20 & 26 & -2 & 0 & 4 & 9 & 12 & 16 & 20 \\
\hline loss (days) & -6 & -4 & 0 & 5 & 9 & 13 & 16 & -6 & -4 & -1 & 3 & 6 & 10 & 12 \\
\hline
\end{tabular}

\section{RCP 6.0}

\section{GFDL-ESM2M}

\begin{tabular}{|c|c|c|c|c|c|c|c|c|c|c|c|c|c|c|}
\hline & \multicolumn{7}{|c|}{$24 \mathrm{~h}$ met } & \multicolumn{7}{|c|}{ 1h met } \\
\hline & P5 & P10 & $\mathrm{P} 25$ & P50 & P75 & P90 & P95 & P5 & $\mathrm{P} 10$ & $\mathrm{P} 25$ & P50 & P75 & P90 & P95 \\
\hline surface temp $\left({ }^{\circ} \mathrm{C}\right)$ & -0.57 & -0.25 & 0.49 & 1.15 & 1.63 & 2.10 & 2.46 & -0.36 & -0.14 & 0.40 & 0.94 & 1.25 & 1.64 & 1.87 \\
\hline bottom temp $\left({ }^{\circ} \mathrm{C}\right)$ & -0.38 & -0.12 & 0.29 & 0.86 & 1.39 & 2.39 & 2.58 & -0.54 & -0.21 & 0.18 & 0.69 & 1.20 & 1.86 & 2.08 \\
\hline whole-lake temp $\left({ }^{\circ} \mathrm{C}\right)$ & -0.15 & 0.18 & 0.57 & 1.15 & 1.72 & 2.06 & 2.22 & -0.10 & 0.07 & 0.39 & 0.88 & 1.31 & 1.55 & 1.64 \\
\hline Schmidt stability $\left(\mathrm{J} \mathrm{m}^{-2}\right)$ & -28.27 & -24.18 & -18.09 & 1.89 & 18.12 & 33.89 & 41.59 & -24.08 & -21.14 & -12.69 & -0.47 & 16.35 & 25.25 & 31.89 \\
\hline thermocline depth (m) & -1.66 & -1.44 & -0.87 & -0.53 & 0.00 & 0.44 & 0.91 & -1.46 & -1.32 & -0.73 & -0.32 & 0.19 & 0.62 & 0.85 \\
\hline duration (days) & -16 & -11 & -3 & 2 & 11 & 17 & 22 & -15 & -8 & -2 & 4 & 10 & 15 & 21 \\
\hline onset (days) & -9 & -8 & -1 & 4 & 10 & 14 & 18 & -8 & -6 & 0 & 5 & 9 & 13 & 17 \\
\hline loss (days) & -14 & -9 & -6 & -2 & 2 & 6 & 7 & -9 & -8 & -4 & -1 & 2 & 5 & 6 \\
\hline
\end{tabular}

HadGEM2-ES

24h met

$1 \mathrm{~h}$ met

\begin{tabular}{lcccccccccccccccc}
\hline & P5 & P10 & P25 & P50 & P75 & P90 & P95 & P5 & P10 & P25 & P50 & P75 & P90 & P95 \\
surface temp $\left({ }^{\circ} \mathrm{C}\right)$ & 0.73 & 1.40 & 2.15 & 3.00 & 4.01 & 4.84 & 5.02 & 0.48 & 0.99 & 1.68 & 2.35 & 3.00 & 3.49 & 3.76 \\
bottom temp $\left({ }^{\circ} \mathrm{C}\right)$ & -0.59 & -0.43 & 0.07 & 0.49 & 1.08 & 1.55 & 1.90 & -0.72 & -0.50 & -0.27 & 0.17 & 0.69 & 1.12 & 1.23
\end{tabular}




\begin{tabular}{|c|c|c|c|c|c|c|c|c|c|c|c|c|c|c|}
\hline whole-lake temp $\left({ }^{\circ} \mathrm{C}\right)$ & 0.39 & 0.96 & 1.50 & 1.98 & 2.89 & 3.28 & 3.51 & 0.22 & 0.65 & 1.15 & 1.54 & 2.05 & 2.35 & 2.49 \\
\hline Schmidt stability $\left(\mathrm{J} \mathrm{m}^{-2}\right)$ & 7.60 & 15.47 & 37.11 & 56.74 & 83.61 & 93.46 & 112.51 & 6.49 & 9.97 & 29.68 & 45.82 & 67.45 & 77.54 & 89.05 \\
\hline thermocline depth (m) & -0.45 & -0.06 & 0.43 & 0.97 & 1.32 & 1.85 & 1.99 & -0.31 & -0.06 & 0.53 & 1.00 & 1.28 & 1.65 & 1.78 \\
\hline duration (days) & 1 & 5 & 15 & 27 & 37 & 44 & 51 & 0 & 4 & 12 & 22 & 30 & 36 & 38 \\
\hline onset (days) & 0 & 1 & 8 & 17 & 22 & 25 & 28 & -1 & 2 & 7 & 14 & 19 & 21 & 22 \\
\hline \multirow[t]{4}{*}{ loss (days) } & -4 & 0 & 5 & 10 & 16 & 21 & 26 & -1 & 0 & 4 & 9 & 13 & 18 & 19 \\
\hline & \multicolumn{14}{|c|}{ IPSL-CM5A-LR } \\
\hline & \multicolumn{7}{|c|}{$24 \mathrm{~h}$ met } & \multicolumn{7}{|c|}{$1 \mathrm{~h}$ met } \\
\hline & P5 & $\mathrm{P} 10$ & $\mathrm{P} 25$ & $\mathrm{P} 50$ & P75 & P90 & P95 & P5 & $\mathrm{P} 10$ & P25 & $\mathrm{P} 50$ & P75 & P90 & P95 \\
\hline surface temp $\left({ }^{\circ} \mathrm{C}\right)$ & 0.49 & 0.96 & 1.50 & 2.69 & 3.52 & 4.25 & 4.55 & 0.33 & 0.72 & 1.07 & 2.08 & 2.63 & 3.15 & 3.45 \\
\hline bottom temp $\left({ }^{\circ} \mathrm{C}\right)$ & -0.51 & -0.21 & 0.26 & 1.12 & 1.67 & 2.52 & 2.76 & -0.51 & -0.29 & 0.12 & 0.78 & 1.27 & 1.91 & 2.11 \\
\hline whole-lake temp $\left({ }^{\circ} \mathrm{C}\right)$ & 0.73 & 1.00 & 1.42 & 2.00 & 2.74 & 3.34 & 3.69 & 0.56 & 0.74 & 1.08 & 1.56 & 2.02 & 2.50 & 2.67 \\
\hline Schmidt stability $\left(\mathrm{J} \mathrm{m}^{-2}\right)$ & -16.36 & -4.48 & 18.04 & 43.96 & 62.09 & 83.42 & 92.30 & -15.94 & -2.04 & 10.72 & 30.78 & 49.06 & 64.78 & 71.12 \\
\hline thermocline depth (m) & -1.28 & -0.80 & -0.34 & 0.29 & 0.75 & 1.05 & 1.14 & -1.15 & -0.68 & -0.20 & 0.40 & 0.75 & 1.03 & 1.18 \\
\hline duration (days) & -7 & 0 & 11 & 21 & 28 & 36 & 44 & -7 & -1 & 9 & 15 & 23 & 29 & 35 \\
\hline onset (days) & -3 & 0 & 5 & 11 & 17 & 21 & 28 & -3 & -1 & 4 & 9 & 14 & 19 & 22 \\
\hline \multirow[t]{4}{*}{ loss (days) } & -7 & -3 & 0 & 5 & 10 & 16 & 18 & -7 & -4 & -1 & 2 & 7 & 10 & 12 \\
\hline & \multicolumn{14}{|c|}{ MIROC5 } \\
\hline & \multicolumn{7}{|c|}{$24 \mathrm{~h}$ met } & \multicolumn{7}{|c|}{$1 \mathrm{~h}$ met } \\
\hline & P5 & P10 & $\mathrm{P} 25$ & P50 & P75 & P90 & P95 & P5 & P10 & $\mathrm{P} 25$ & P50 & P75 & P90 & P95 \\
\hline surface temp $\left({ }^{\circ} \mathrm{C}\right)$ & 0.75 & 0.89 & 1.46 & 2.15 & 3.01 & 3.66 & 4.04 & 0.57 & 0.68 & 1.17 & 1.68 & 2.37 & 2.85 & 3.05 \\
\hline bottom temp $\left({ }^{\circ} \mathrm{C}\right)$ & -0.31 & 0.04 & 0.48 & 0.98 & 1.56 & 1.98 & 2.27 & -0.42 & -0.21 & 0.20 & 0.79 & 1.19 & 1.57 & 2.12 \\
\hline whole-lake temp $\left({ }^{\circ} \mathrm{C}\right)$ & 0.62 & 0.81 & 1.26 & 1.81 & 2.46 & 3.00 & 3.13 & 0.44 & 0.58 & 0.97 & 1.36 & 1.92 & 2.23 & 2.43 \\
\hline Schmidt stability $\left(\mathrm{J} \mathrm{m}^{-2}\right)$ & -3.28 & 0.53 & 12.21 & 22.98 & 45.52 & 63.24 & 75.29 & -6.66 & -1.07 & 9.20 & 19.17 & 36.39 & 53.98 & 57.02 \\
\hline thermocline depth (m) & -0.94 & -0.63 & -0.19 & 0.22 & 0.64 & 0.98 & 1.21 & -0.54 & -0.35 & 0.05 & 0.33 & 0.78 & 1.15 & 1.33 \\
\hline duration (days) & -6 & 4 & 9 & 16 & 24 & 34 & 39 & -4 & 1 & 7 & 15 & 19 & 27 & 32 \\
\hline onset (days) & -1 & 2 & 9 & 12 & 17 & 23 & 28 & -3 & 1 & 7 & 11 & 14 & 20 & 22 \\
\hline loss (days) & -4 & -2 & 1 & 5 & 9 & 12 & 17 & -4 & -3 & 0 & 4 & 6 & 9 & 12 \\
\hline
\end{tabular}

